\title{
Design considerations for primary control in multi-terminal VSC-HVDC grids
}

\author{
Q1 , Catalin Gavriluta ${ }_{\wedge}^{\mathrm{a}, *}$, Ignacio Candela ${ }_{\wedge}^{\mathrm{a}},{ }_{\Lambda}$ Costantino Citro ${ }_{\wedge}^{\mathrm{a}}$, Alvaro Luna ${ }_{\wedge}^{\mathrm{a}}$, \\ Pedro Rodrigueza ${ }_{\wedge}^{\mathrm{a}, \mathrm{b}}$ \\ a Renewable Electrical Energy Systems (SEER), Technical University of Catalonia (UPC), Rambla de Sant Nebridi 22, 08222 Terrassa, Spain \\ ${ }^{\mathrm{b}}$ Abengoa Research, C/Energía Solar 1, Palmas Altas, 41014 Seville, Spain
}

\section{A R T I C L E I N F O}

\section{Article history:}

Received 23 May 2014

Received in revised form 1 December 2014

Accepted 19 December 2014

Available online $\mathrm{xxx}$

\section{Keywords:}

Multi-terminal HVDC

Droop control

Oscillation damping

Capacitor size

Q2 Hierarchical control

\begin{abstract}
A B S T R A C T
Multi-terminal dc networks based on voltage source converters (VSC) are the latest trend in dc-systems; the interest in the area is being fueled by the increased feasibility of these systems for the large scale integration of remote offshore wind resources. Despite the active research effort in the field, at the moment issues related to the operation and control of these networks, as well as sizing are still uncertain. This paper intends to make a contribution in this field by analyzing the sizing of droop control for VSC together with the output capacitors. Analytical formulas are developed for estimating the voltage peaks during transients, and then it is shown how these values can be used to dimension the dc-bus capacitor of each VSC. Further on, an improved droop control strategy that attenuates the voltage oscillations during transients is proposed. The proposed methods are validated on the dc-grid benchmark proposed by the CIGRE B4 working group. Starting from the structure of the network and the power rating of the converters at each terminal, the output capacitors and the primary control layer are designed together in order to ensure acceptable voltage transients.
\end{abstract}

(C) 2014 Published by Elsevier B.V.

\section{Introduction}

For the past century ac networks have been established as the standard technology for electrical power transmission systems. However, the dc technology has not disappeared completely from this picture. The capability of dc systems to transmit higher power over longer distances, the possibility of interconnecting asynchronous networks, and their high efficiency have maintained Q3 the interest of both industry and academia. Even though historically dc generators and mercury arc rectifiers have been used for dc transmission, for the past five decades, HVDC applications were almost exclusively built using line commutated converters (LCC) based on thyristors. More than 145 installations with power ratings ranging up to $7000 \mathrm{MW}$ have been commissioned until 2013 in over 40 countries worldwide, and 40 new projects are planned to be finished before 2020 [1]. In parallel with LCCs a new technology for HVDC applications has emerged in 1990s, with a first $50 \mathrm{MW}$ installation in Gotland, Sweden in 1999. This new technology uses voltage source converters (VSC) based on IGBTs.

\footnotetext{
* Corresponding author. Tel.: +34 937398549.

E-mail address: catalin.gavriluta@upc.edu (C. Gavriluta).
}

While LCC is a mature technology that is successfully used in applications transferring very large amounts of power with high efficiency, it has the drawbacks of drawing considerably amounts of reactive power and generating a large number of low-harmonics, hence it requires a strong connection to the ac-network [2]. Moreover, the operation and control of LCC dc networks with more than two terminals has proved to be extremely difficult.

VSCs, on the other hand, can connect to very weak ac-grids. They can provide reactive power and have black start capability. The smaller filters and compensators reduce considerably the overall footprint of the station, making it ideal for offshore platforms and dense urban environments. Higher losses and smaller power ratings used to be the main disadvantage of the VSC, but advances in multi-modular converters (MMC) are tackling this issue [3].

The fast advances in power electronics, together with the challenges raised by the integration of distributed generation into the modern distribution network, has drawn a lot of attention towards VSC multi-terminal dc (MTDC) networks. Daring projects as the North Sea Supergrid, which propose to use such a network for integrating the wind power resources of the North Sea in the mainland grids, have fueled the research in the area for the past few years [4-9]. Even though the topic of HVDC for the integration of offshore wind farms is the most approached subject in the area, other authors have started to analyze the benefits of MTDC grids in other 
applications, such as railway electrification [10] or microgrids with mixed sources of energy [11],[12].

One of the main challenges of MTDC networks is related to their control and operation. While several application-specific approaches can be found in the literature [13-16], it is starting to become clear that the most generic, scalable, and reliable control method is a hierarchical approach, similar to the one used in ac networks, as suggested by [17-19]. In this hierarchical approach, the primary layer is decentralized and independent of any communication link. Several decentralized control methods for MTDC grids are identified in [20]. From these methods the droop control seems to be the most common voltage control strategy, being used either exclusively[21] either in combination with other voltage control strategies [7]. Meanwhile, the secondary control is centralized and regulates the voltage profile of the network, as well as the basic power flow, by making use of low-bandwidth communication [22-24].

Even though the interest towards MTDC networks is exponentially growing, at the moment there is no standard test-platform for investigating their operation. The only resource found in the literature in this sense is the work of the CIGRE B4 DC work-group [25], who is lately pursuing an active work in characterizing and sizing a test bench for MTDC systems. However, there is still work to be done in this direction.

One of the most important parameters of VSCs connected to a MTDC network is the size of the dc-bus capacitor. Standard methods for designing power converters address the sizing of the dc-bus capacitor by taking into account considerations related to voltage ripple [26] or controller dynamics [27,28]. However, when set in the context of a dc grid, the capacitor becomes the inertial element of the network and, more than affecting the time constants of the primary control, it also influences the network dynamics and determines how strong is the network. While very large capacitors would be desirable from the inertial point of view, the large short-circuit currents impose difficult requirements for the protection system.

This paper proposes a method for sizing the dc-bus capacitors by analyzing the amplitude of the voltage overshoot during transients. For this, analytical methods for estimating these overshoots are developed and tested on a five terminal dc network presented in the CIGRE B4 DC Grid Test System. Having an estimate of the voltage overshoot during transients is also required for secondary control. Most of the methods presented in the literature for optimal power flow in MTDC networks $[29,30]$ bind the voltage of one generatorbus to a value equal to the maximum voltage, while the voltages at the other buses are imposed by the power flows that they have to accommodate. This method raises serious concerns regarding the vulnerability of the network during sudden load changes, as it does not allow any headroom for transient overvoltages. This can be avoided if the maximum voltage at which the generating bus is bound is computed by taking into account the voltage overshoots that can occur in the network at each moment.

Further on, based on the analysis of voltage transients an improved droop control method is proposed. The proposed control strategy offers additional voltage damping during transients without affecting the steady state behavior.

The paper is organized as follows: Section 2 presents the mathematical tools for computing the overshoot of typical transfer functions. Section 3 shows how the mathematical approach previously presented can be used for analyzing the dynamics of the dc voltage droop control. Section 4 analyzes the impact of the connecting cable on the voltage dynamics. Section 5 proposes an enhanced droop control method for attenuating the voltage oscillations during transients. The proposed methods for sizing the parameters of the system, as well as the proposed droop control with damping are validated by simulation in Section 6. Finally, Section 7 presents the conclusions of the present work.

\section{Mathematical prerequisites}

The method for sizing the capacitors of the dc-network that is proposed in this paper relies on the estimation of the voltage overshoot during transients generated by the connection or disconnection of loads. This approach is proposed in order to ensure that the voltage will not reach values that might damage the converters during sudden load changes.

As it will be seen in the following sections, the transfer functions to be studied can be approximated by second order systems or, in some cases, second order systems with a zero. Therefore, we will start by providing analytical formulas for calculating the overshoot of these systems to a step change.

\subsection{Second order system}

$H_{2}(s)$ in (1) is the canonical form of a second order system, where $\omega_{n}$ is the natural oscillation frequency of the system and $\xi$ is the damping of the system. Considering the under-damped case, $\xi<1$, the time response of the system, $y_{2}(t)$, to a step change can be calculated as shown in (2).

$$
\begin{aligned}
H_{2}(s) & =\frac{\omega_{n}^{2}}{s^{2}+2 \xi \omega_{n}+\omega_{n}^{2}} \\
y_{2}(t) & =\mathcal{L}^{-1}\left\{\frac{1}{s} \cdot H_{2}(s)\right\} \\
& =1-e^{-\xi \omega_{n} t}\left[\cos \left(\omega_{d} \cdot t\right)+\frac{\xi}{\sqrt{1-\xi^{2}}} \sin \left(\omega_{d} \cdot t\right)\right] \\
\omega_{d} & =\omega_{n} \cdot \sqrt{1-\xi^{2}}
\end{aligned}
$$

In order to get the maximum value of $y_{2}(t)$, we can set its derivative to zero to obtain the time value $t^{p k}$ at which the peak of $y_{2}$ occurs. Afterwards, by evaluating $y_{2}$ at $t^{p k}$, one can obtain the peak overshoot, $y_{2}^{p k}$ as shown in (3).

$$
\begin{aligned}
\left.\frac{d y_{2}(t)}{d t}\right|_{t=t^{p k}} & =0 \\
t^{p k} & =\frac{\pi}{\omega_{d}} \\
x_{2}^{p k} & =1+e^{-\pi \cdot \frac{\xi}{\sqrt{1-\xi^{2}}}}
\end{aligned}
$$

\subsection{Second order system with a zero}

The same methodology applied for calculating the overshoot of second order systems can be applied for second order systems with a zero. The transfer function of such a system is shown in (4).

$H_{20}(s)=\frac{\omega_{n}^{2} \cdot(a \cdot s+1)}{s^{2}+2 \xi \omega_{n} s+\omega_{n}^{2}}=a \cdot s \cdot H_{2}(s)+H_{2}(s)$

When calculating the transient response to a step change in time domain, one can see it as the sum of the response of a second order system and its derivative scaled by the $a$ term, as seen in (5).

$$
\begin{aligned}
y_{20}(t) & =\mathcal{L}^{-1}\left\{\frac{1}{s} \cdot H_{20}(s)\right\} \\
& =a \cdot \frac{d y_{2}(t)}{d t}+y_{2}(t) \\
& =1-e^{-\xi \omega_{n} t}\left[\cos \left(\omega_{d} \cdot t\right)+\beta \sin \left(\omega_{d} \cdot t\right)\right] \\
\beta & =\frac{\xi}{\sqrt{1-\xi^{2}}}-a \cdot \omega_{d}-\frac{\xi^{2}}{\sqrt{1-\xi^{2}}} \cdot a \cdot \omega_{n}
\end{aligned}
$$



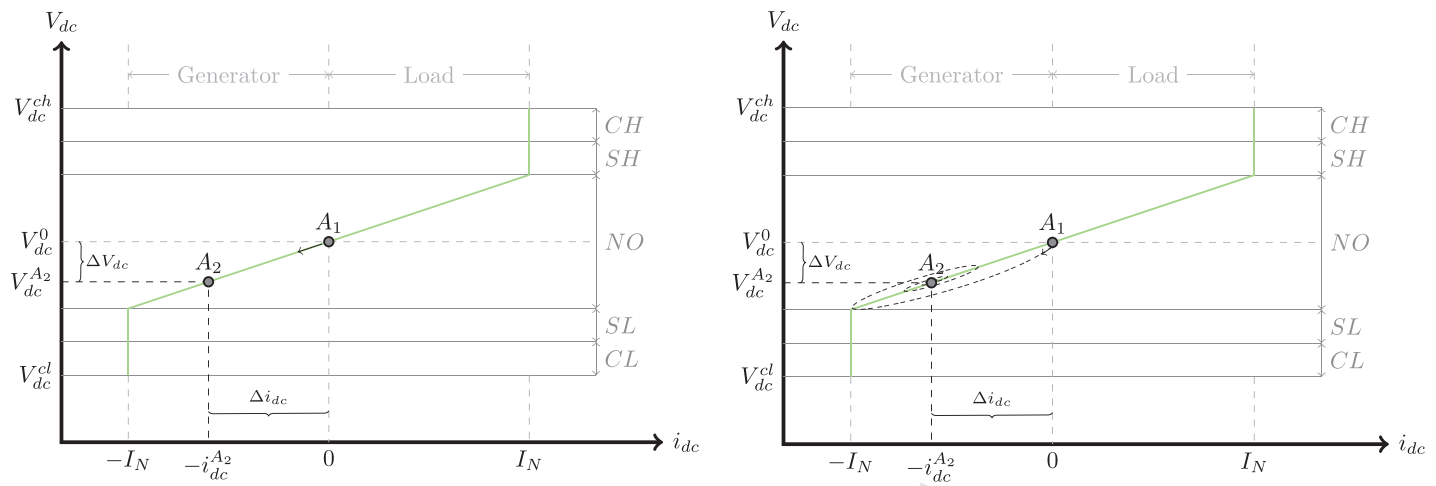

Fig. 1. DC-voltage operating bands and droop characteristic ${ }_{\wedge}$

$$
\begin{aligned}
\left.\frac{d y_{20}(t)}{d t}\right|_{t=t^{p k}} & 0 \\
t^{p k} & =\frac{1}{\omega_{d}} \cdot\left[\tan ^{-1}\left(-\frac{a \cdot \omega_{n}^{2}}{\omega_{d}+\xi \cdot \omega_{n} \cdot \beta}\right)+\pi\right] \\
x_{20}^{p k} & =1-e^{-\xi \omega_{n} t^{p k}} \cdot\left(\cos \left(\omega_{d} \cdot t^{p k}\right)+\beta \cdot \sin \left(\omega_{d} \cdot t^{p k}\right)\right)
\end{aligned}
$$

\section{DC voltage droop control}

The concept of droop control for the dc voltage of a MTDC network is always described in the literature by its steady state behavior, i.e., a linear relationship between deviations in voltage and deviations in the injected power or current. Such an example is shown here in Fig. 1a, based on our previous work presented in [31]. Here, a bidirectional VSC (i.e., a VSC that can operate both as a load and as a generator) reacts to a decrease in the voltage $\Delta V_{d c}$ by increasing the generated current with $\Delta i_{d c}$.

We can see that there are several voltage intervals defined around the nominal voltage $V_{d c 0}$. The normal operation (NO) interval is chosen so that the bus-voltages of the network, corresponding to the possible power flow scenarios, are included inside this operation band. Further on, the safety-limit intervals, namely safety low $(\mathrm{SL})$ and safety high $(\mathrm{SH})$, are reserved for transients that deflect the voltage of the network from the NO band for a short period of time. Finally the critical voltage intervals correspond to highly overloaded scenarios, i.e., the critical low (CL) operating band, and highly overcharged scenarios, i.e., the critical high $(\mathrm{CH})$ operating band.

The definition of the operating voltage bands and how they relate to droop control is described in details in [31], but the article provides no method for dimensioning these operating bands for a given system, nor a solution for the reciprocal: having selected the height of the operating bands, the parameters of the system should be dimensioned so that voltage intervals are always satisfied.

The sizing of the NO band can be approached by analyzing the steady state interaction between the conductance matrix of the grid and the gains of the droop control using methods proposed in [21]. Meanwhile, the critical bands will be limited by the minimum and maximum operating voltages $V_{d c}^{c l}$ and $V_{d c}^{c h}$, that are typically given by the hardware limitations of the elements connected to the grid. For example, $V_{d c}^{c l}$ can be considered the voltage level for which the converters go into over-modulation, while $V_{d c}^{c h}$ can be imposed by the peak rating of the power electronics used in the converters. However, between the NO band and the critical bands,

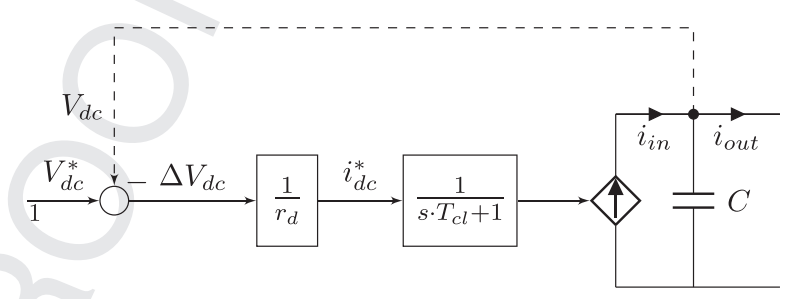

Fig. 2. Block diagram of DC-bus voltage controlled by droop.

the safety bands have to be assessed. This aspect is approached in the next sections by analyzing the voltage overshoots that occur in the network during transients and the parameters that influence them.

\subsection{Droop control dynamics}

The change in the operating point of the converter is showed as sliding along the droop curve in Fig. 1. However, this is not the response expected from a real system. In reality, due to overshoots and oscillations in both voltage and current, the trajectory followed by the operating point of the converter will more likely look as the one presented in Fig. 1b. Understanding what parameters have an important effect on these oscillations will help in sizing the system in order to reduce its oscillatory behavior.

The control structure of the dc-bus voltage considered for this analysis is composed of two loops: an internal current loop and an external voltage loop. As shown in Fig. 2, the current loop is considered to have perfect closed loop dynamics and is approximated with a first order system with time constant $T_{c l}$. The outer voltage loop is controlled using a droop strategy, with an equivalent droop resistance $r_{d}$.

Fig. 3 shows the typical evolution of the current injected by the converter and of the dc voltage after a step change in the load current, $i_{\text {out }}$. $\mathrm{Eq}_{\lambda}$ (7) shows the relationship between the voltage reference, the load/disturbance current $i_{\text {out }}$, and the injected current $i_{\text {in }}$; with the corresponding transfer functions being defined in (8) and (9).

$i_{\text {in }}=v_{d c}^{*} \cdot H_{* u}+i_{\text {out }} \cdot H_{d u}$

$H_{* u}=\frac{C \cdot s}{C \cdot T_{c l} \cdot r_{d} \cdot s^{2}+C \cdot r_{d} \cdot s+1}$

$H_{d u}=\frac{1}{C \cdot T_{c l} \cdot r_{d} \cdot s^{2}+C \cdot r_{d} \cdot s+1}$

The effect of the reference changes on the injected current is not so critical as the effect of the disturbance since additional filters can be mounted on the input path in order to smooth any sudden change in reference. As seen in (9) the transfer function from the 


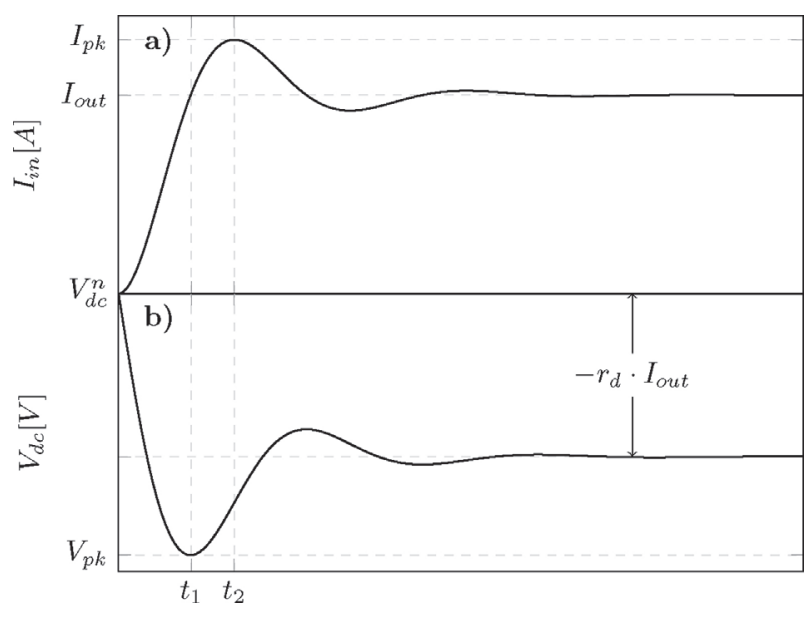

Fig. 3. Evolution of the $(a)$ current injected by the VSC and $(b)$ dc voltage to a step in the load current.

disturbance current to the actual injected current is of second order and can be written in the canonical form with the terms presented in (10).

$$
\begin{gathered}
\xi=\sqrt{\frac{r_{d} \cdot C}{4 T_{c l}}} \\
\omega_{n}=\sqrt{\frac{1}{r_{d} \cdot C \cdot T_{c l}}}
\end{gathered}
$$

There are some important aspects that can be observed from these expressions. If the response time of the current loop is fast, i.e., $T_{C l}$ very small, then the response is approximately the one of a first order system with a time constant equal to $r_{d}$.C. Also, the damping of the system can be improved either by making the current loop faster, therefore decreasing $T_{c l}$, either by increasing $r_{d}$ or $C$.

As seen in Fig. 3 the current response will experience a peak value, $I_{p k}$. Using the procedure presented in the previous section, for a unit step in $i_{\text {out }}$, the peak current injected by the converter is obtained as shown in (11). For safety reasons, this value has to be smaller than the peak rating of the converter.

$I_{p k}=1+e^{-\pi \cdot \sqrt{\frac{r_{d} \cdot C}{4 T_{C l}{ }^{-r} d \cdot C}}}$

Further on, by taking the transfer functions of the systems interacting in Fig. 2 we can determine the expression of the output voltage as shown in (12). We can see that the effect of the disturbance on the dc voltage is that of a second order system with a zero. As previously explained, a fast current loop would transform the system into a first order system with the time constant imposed by $r_{d}$ and $C$.

$v_{d c}=v_{d c}^{*} \cdot H_{* y}(s)+i_{o u t} \cdot H_{d y}(s)$

$H_{* y}(s)=\frac{1}{C \cdot T_{c l} \cdot r_{d} \cdot s^{2}+C \cdot r_{d} \cdot s+1}$

$H_{d y}(s)=-\frac{r_{d} \cdot\left(T_{c l} \cdot s+1\right)}{C \cdot T_{c l} \cdot r_{d} \cdot s^{2}+C \cdot r_{d} \cdot s+1}$

As shown in Fig. 3 the steady state voltage offset is equal to the product between the droop resistance and the load current. Meanwhile, the peak value of the voltage during transients, $V_{p k}$, can be calculated by making use of the formulas presented in the previous section. In the case of a sudden decrease of load, $V_{p k}$ will be larger than the nominal dc voltage, but it should not overpass the rating of the converter.

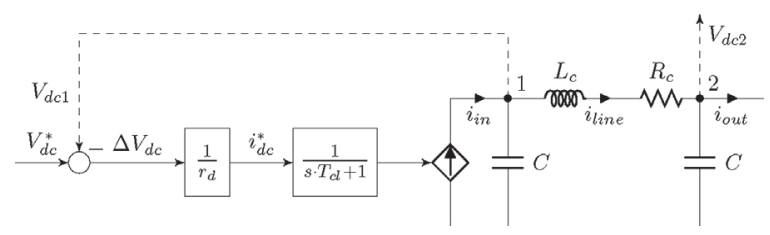

Fig. 4. Block diagram of DC-bus voltage controlled by droop with the load connected at the end of cable.

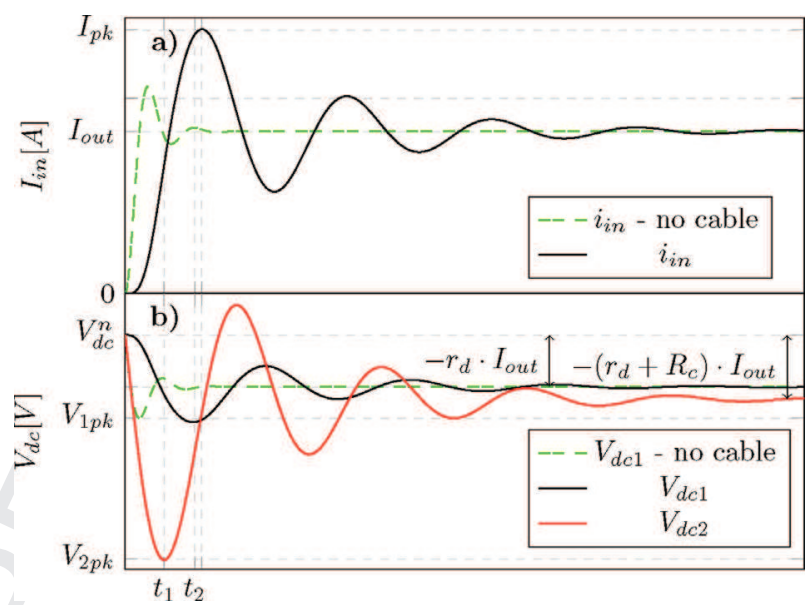

Fig. 5. Evolution of the (a) current injected by the VSC and (b) dc voltage at the two ends of the cable after a step in the load current.

\section{Effect of the connecting-cable on droop control dynamics}

A more critical scenario that has to be analyzed is when the load is connected to the converter through a long cable or overhead line as shown in Fig. 4. Here, an equivalent $\pi$-model is used for the cable, and the shunt capacitors are lumped together with the dcbus capacitors of the two converters connected at each end of the line.

Fig. 5 shows a typical evolution of the current injected by the converter as well as of the voltages at the two ends of the cable. The previous case, in which the cable was not included in the analysis, is added to the figure with a dotted line for comparison. It can be seen that the presence of the cable results in larger transients. The largest voltage overshoot will appear at the terminal where the load change occurred. For the case presented in Fig. 5, this is the overshoot of the voltage at terminal 2, namely $V_{2 p k}$.

The previously used method for calculating the overshoots in the system cannot be applied for obtaining $V_{2 p k}$ because the transfer function from $i_{\text {out }}$ to $v_{2}$, for the complete system, is of a higher order. However, a good approximation can be obtained by considering terminal 1 as a constant voltage source, as shown in Fig. 6. Fig. 7 shows the evolution of the voltage at terminal 2 after a step change in the load, while considering different values of the droop resistance. It can be seen that, while the droop resistance has a considerable effect on the attenuation of the voltage oscillations, it has almost no effect on the first peak. Therefore, we can assume that $V_{2 p k}$ is independent of the droop resistance, and we can approximate it with the value obtained by considering the system connected to a constant voltage source.

For the system presented in Fig 6, the relationship between $v_{2}$ and $i_{\text {out }}$ is described by (15) and (16). As it can be seen, the equivalent transfer function corresponds to a second order with an added 


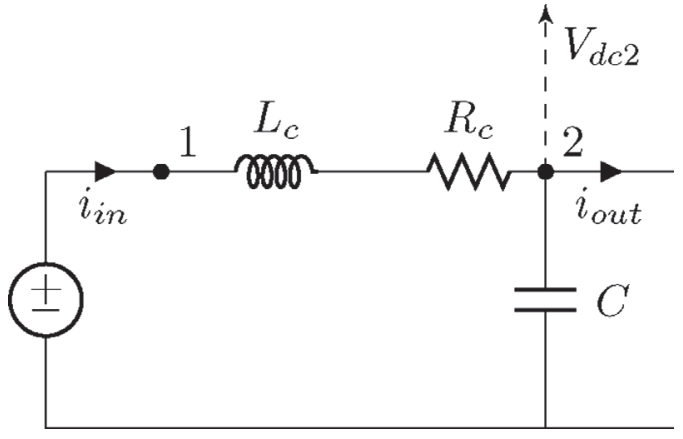

Fig. 6. Perturbation at the end of a cable connected to a voltage source $\wedge$

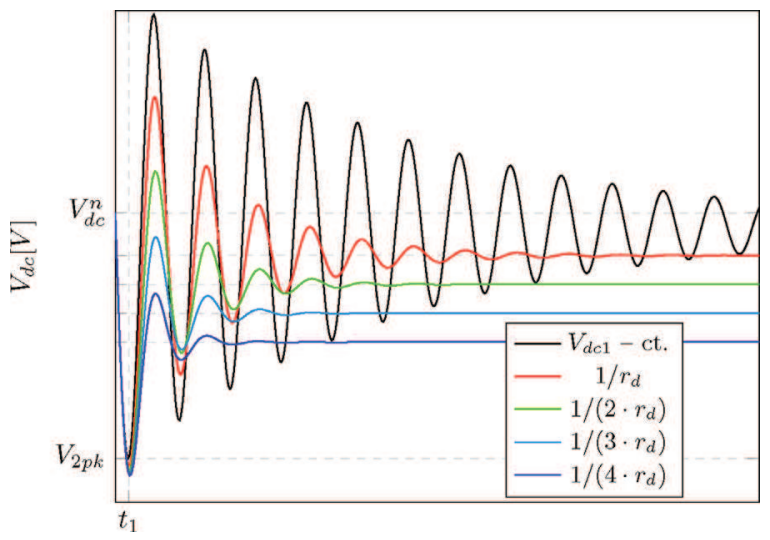

Fig. 7. Evolution of the dc-bus voltage $V_{d c 2}$ for different values of droop resistance used in the voltage at terminal 1 .

zero. For this type of transfer function, the methodology presented in Section 2 can be used in order to calculate its overshoot.

$V_{d c 2}=\quad i_{\text {out }} \cdot H_{c}(s)$

$H_{c}(s)=k \cdot \frac{\omega_{n}^{2} \cdot(a \cdot s+1)}{s^{2}+2 \xi \omega_{n} s+\omega_{n}^{2}}$

$a=\frac{L_{c}}{R_{C}} \quad, \quad \omega_{n}=\sqrt{\frac{1}{L_{C} \cdot C}}$

$k=R_{c}, \quad \xi=\sqrt{\frac{R_{c}^{2} \cdot C}{4 \cdot L_{C}}}$

It has to be underlined that the independence of $V_{2 p k}$ on $r_{d}$ can not be considered true for the general case. Very large values for $r_{d}$, as say $r_{d} \rightarrow \infty$, will reduce the voltage to zero as the converter at terminal 1 will not contribute to the regulation of voltage. Also, very small capacitors at terminal 1 , or very large $T_{c l}$ will have a negative impact on the first overshoot of the voltage. Basically, in order for this approximation to hold true, the dynamics of the voltage control at terminal 1 have to be negligible when compared with the dynamics of the cable.

Also, since the proposed method relies on the values of the cable impedance, in order to cope with variations in $R_{c}$ or $L_{c}$ due to heating or tripping of parallel lines, one could consider a worstcase scenario in the planning phase in order to ensure larger safety limits.

\section{Droop control with dynamic damping}

As mentioned earlier, the value of the droop resistance has a considerable effect on the attenuation of the voltage oscillations. A larger droop resistance will damp the oscillations faster, but it will

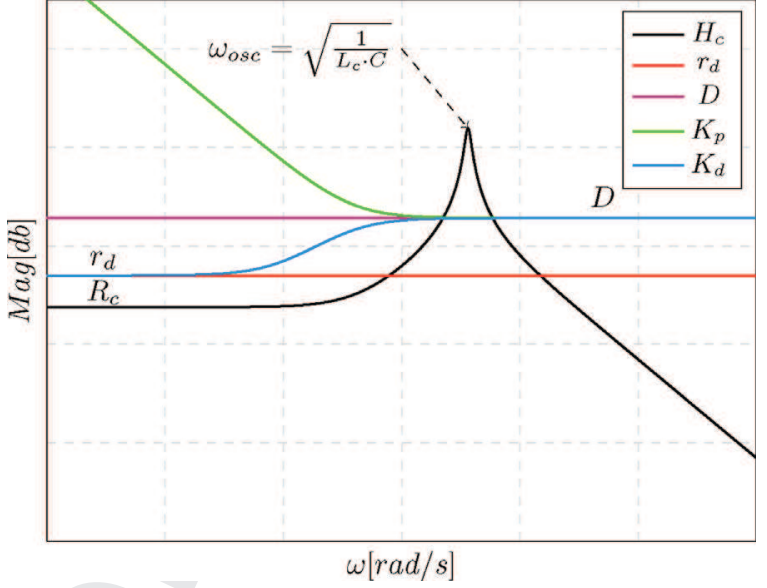

Fig. 8. Design of the droop control with damping.

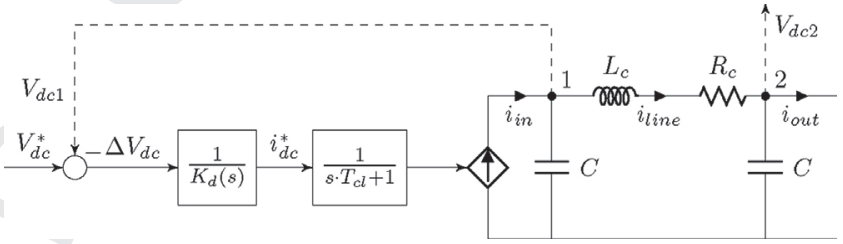

Fig. 9. Block diagram of DC-bus voltage controlled by dynamic droop.

also impose a larger steady-state voltage deviation. On the other hand, a smaller droop resistance will provide less damping, but has the advantage of reduced steady-state voltage deviations. A better option is to combine the advantages of the two into a frequency dependent characteristic that has a small gain at steady state and a larger gain at the frequencies where oscillations are expected.

Fig. 8 displays the magnitude characteristic of a typical $H_{c}(s)$, as defined in (15). At steady-state, only the influence of the cable resistance is notable, but as the frequency increases, the parasitic inductance of the cable starts to resonate with the capacitor at the output of the converter, creating a peak in magnitude at $\omega_{o s c}$, defined in (17).

$\omega_{o s c}=\frac{1}{\sqrt{L_{C} \cdot C}}$

A constant droop resistance, $r_{d}$, will offer the same amount of damping over the entire frequency range as seen in Fig. 8. However, a dynamic droop resistance $K_{d}$, as defined in (18), will have the same steady-state behavior as $r_{d}$, but it will provide a larger damping at the resonating frequency.

$K_{d}(s)=r_{d} \frac{1+\frac{s}{\omega_{z}}}{1+\frac{s}{\omega_{p}}}$

The standard droop control is replaced with $K_{d}$, as shown in Fig. 9, and the response of the system is shown Fig. 10. The dotted lines ${ }_{-}$displayed for comparison ${ }_{-}$are the responses of the droop controller, previously shown in Fig. 5. The solid lines are the responses obtained by using $K_{d}$. Several aspects should be pointed out. Firstly, $K_{d}$ can also be seen as a damper on the current dynamics; the current response to high frequency perturbation is slowed down. In this way the peak overshoot of the current is considerably reduced, as seen in Fig. 10a. As the current response is slower, the voltage at terminal 1 presents a much larger peak compared with the previous case and the voltage at terminal 2 also presents a slightly larger overshoot. In terms of damping, it can be clearly noted that the oscillations are considerably attenuated by using $K_{d}$. 


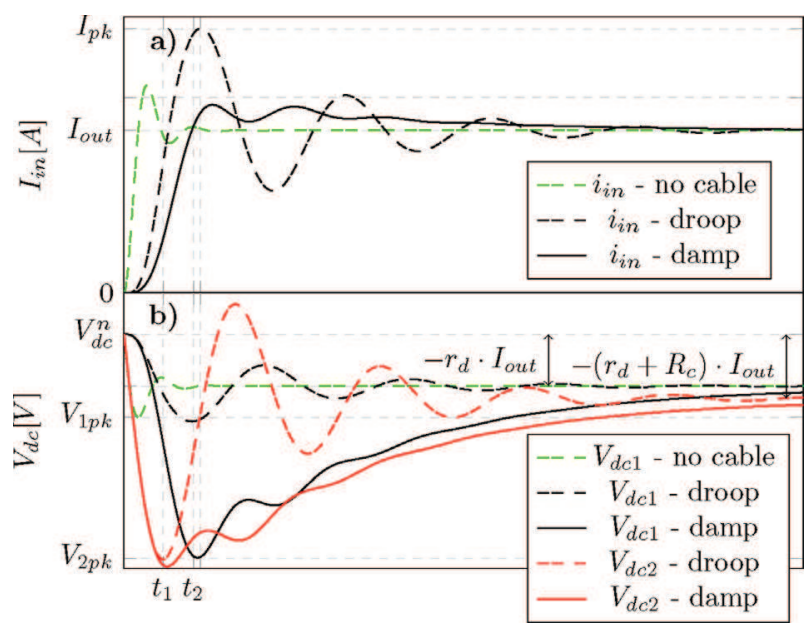

Fig. 10. Effect of the damping controller on the (a) current injected by the VSC and (b) dc-voltage at the two terminals.

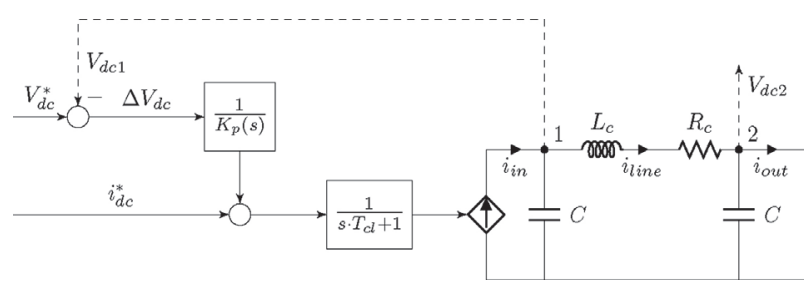

Fig. 11. Block diagram of constant current element with added oscillation damping controller.

In order to tune $K_{d}$, one needs to calculate $\omega_{z}$ and $\omega_{p}$ based on (19) and (20).

$\omega_{p}=\frac{\omega_{o s c}}{10}$

$\omega_{z}=\frac{r_{d}}{D} \omega_{p}$

We choose the frequency of the pole, $\omega_{p}$, so that the gain of $K_{d}$ flattens out one decade before the oscillation frequency. The frequency of the zero, $\omega_{z}$, is chosen based on the value of the steadystate droop resistance, $r_{d}$, and the value of the damping resistance $D$. Choosing the frequency for the pole and zero in this fashion, ensures that at the resonating frequency the gain of $K_{d}$ will be equal to $D$. It can be seen that there is a trade-off between $D$ and $\omega_{z}$ which can be seen as a trade-off between the attenuation level and the increase in the voltage overshoot. A very large $D$ will provide very good damping, but it will considerably reduce $\omega_{z}$ and consequently, it will slow the current dynamics even further, thereby resulting in a large voltage drop. On the other hand, a small damping factor will have a small effect on the oscillation. Therefore, the best tradeoff between damping performance and voltage drop is obtained by choosing $D$ as shown in (21).

$D=\sqrt{\frac{L_{c}}{C}}$

The proposed controller offers additional damping for the elements of a network that are operated in droop mode. However, a similar damping controller can be designed for the nodes that are in constant power control. Having the voltage error as input, a damping element $K_{p}$, defined in (22), can be added as seen in Fig. 11 . The only difference between $K_{d}$ and $K_{p}$ is that at steadystate the latter has infinite gain. An important aspect that has to be taken into consideration when choosing $\omega_{p}$ and $D$ for converters working in constant power mode, it is the amount of inertia of the

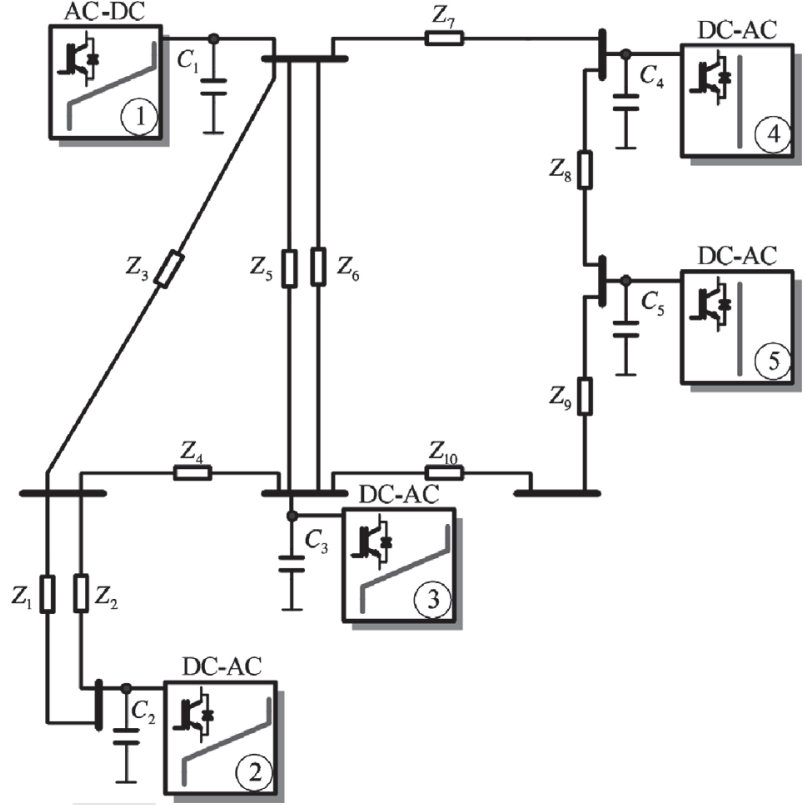

Fig. 12. Five terminal HVDC grid proposed by the CIGRE B4 working group ${ }_{\triangle}$

Table 1

Data for the connecting lines proposed in [25],

\begin{tabular}{llll}
\hline & $\mathrm{R}[\Omega / \mathrm{km}]$ & $\mathrm{L}[\mathrm{mH} / \mathrm{km}]$ & $\mathrm{C}[\mu \mathrm{F} / \mathrm{km}]$ \\
\hline Overhead line (OHL) & 0.0114 & 0.9356 & 0.0123 \\
Cable (CBL) & 0.0095 & 2.1120 & 0.1906 \\
\hline
\end{tabular}

primary source of energy to which the converter is connected. During transients, a too small damping factor $D$ could result in sudden large deviations from the initial power reference; this deviation can not be supported by elements with no inertia.

$K_{p}(s)=\frac{1+\frac{s}{\omega_{p}}}{\frac{s \cdot D}{\omega_{p}}}$

\section{Study case}

\subsection{Network topology}

As a study case for demonstrating the proposed concepts we selected a five terminal dc network proposed by the CIGRE B4 DC working group, and reproduced here in Fig. 12. In this network the converters at terminals 1,2 , and 3 (T1, T2, and T3) are droop controlled and link the dc-network with neighboring ac systems. In the original proposal of this system converters 4 and 5 are used as interfaces for two offshore wind power plants, therefore they will be operated as constant power elements in our analysis.

Since part of the network is considered to be offshore, some of the interconnecting lines are submarine cables $(\mathrm{CBL})$ while the others are overhead lines (OHL). The parameters per unit of length proposed in [25] for the two types of lines are presented in Table 1. In our analysis we used an equivalent $\pi$-model for the connecting lines and cables. The parameters of the models used for each line are shown in Table 2 . In ac-systems the equivalent $\pi$-model is only accepted for cables with lengths up to $50-80 \mathrm{~km}$. However, in the dc-system that we are investigating, the capacitor at the output of the converter is expected to be considerably larger than the cable capacitance. Our simulations showed very little difference in the dynamic response of a cable modeled by a single $\pi$-section and one modeled by multiple sections when the cable is terminated with the large capacitors required for the operation of the VSC. 
Table 2

Parameters for the equivalent $\pi$-model $\wedge$

\begin{tabular}{lcccccc}
\hline & $Z_{1,2}$ & $Z_{3}$ & $Z_{4}$ & $Z_{5,6}$ & $Z_{7,8,10}$ & $Z_{9}$ \\
\hline$R_{\pi}[\Omega]$ & 3.42 & 5.70 & 2.28 & 4.56 & 1.90 & 2.85 \\
$L_{\pi}[\mathrm{H}]$ & 0.28 & 0.46 & 0.19 & 0.37 & 0.42 & 0.63 \\
$C_{\pi}[\mu \mathrm{F}]$ & 1.85 & 3.08 & 1.23 & 2.46 & 19.06 & 28.60 \\
len. $[\mathrm{km}]$ & 300 & 500 & 200 & 400 & 200 & 300 \\
Type & OHL & OHL & OHL & OHL & CBL & CBL \\
\hline
\end{tabular}

Table 3

Rating of the converters connected at the five terminals

\begin{tabular}{lccccc}
\hline Terminal: & T1 & T2 & T3 & T4 & T5 \\
\hline$P_{n}[\mathrm{MW}]$ & 2400 & 2400 & 2400 & 800 & 1600 \\
$V_{d c}^{0}[\mathrm{kV}]$ & \pm 400 & \pm 400 & \pm 400 & \pm 400 & \pm 400 \\
Type & Droop & Droop & Droop & ct. P & ct. P \\
\hline
\end{tabular}

Table 3 presents the rating of the converters connected at the five terminals of the network. T1, T2, and T3 are rated at $2400 \mathrm{MW}$, equal to the maximum aggregated power of T4 and T5. The operating voltage for the network, $V_{d c}^{0}$, is set at $\pm 400 \mathrm{kV}$. The three droop controlled converters have been modeled as shown in Fig. 2, and a similar approach has been used for modeling the constant power nodes.

\subsection{Primary control}

The achievable speed of the current loop is an important parameter in the sizing of the system and can vary substantially depending on the topology of the converter and the control structure. Classic two level converters have to reduce their switching frequency as the power rating increases due to the switching losses and this unavoidably reduces the speed of the current loop. On the other hand, modern multi-modular converters will respond faster due to the reduced filter size. For our analysis we imposed the bandwidth of the current loop to be at around $200 \mathrm{~Hz}$ as it can be considered achievable also for converters switching at slower frequency.

The remaining parameters of the system are the values of the five dc capacitors, $C_{1 . .5}$, and the values of the droop resistances.

Following the definition of the voltage operating bands from Section 3, for our analysis we have chosen $N O=V_{d c}^{0} \cdot 10 \%$, and a width of $5 \%$ for the rest of the operating bands. In this manner, the maximum allowed deviation from $V_{d c}^{0}$ is $15 \%$.

The size of $N O$ will have a direct influence on the maximum droop resistance that can be used. Basically, the series combination of the droop resistance and the equivalent resistance of the lines should be smaller than $R_{\max }=N O / I_{n}$, where $I_{n}$ is the nominal current of the converter.

For the network presented in Fig 12, the equivalent cable resistance seen from the three droop-controlled terminals can be approximately calculated as: $R_{e 1}=0.8971 \Omega, R_{e 2}=3.3386 \Omega$, and $R_{e 3}=1.0205 \Omega$. For the chosen $N O, R_{\max }$ is calculated as $13.33 \Omega$, therefore, in order to respect the voltage limits, the droop resistance $r_{d}$ is chosen equal to $10 \Omega$ for the converters at terminals 1,2 , and 3.

\subsection{Sizing of output capacitors}

For sizing the capacitors we are going to make use of the analysis described in Section 4. From the way we defined the operating voltage bands, the maximum voltage deviation is $15 \%$. Therefore, the size of the capacitors has to be calculated so that the voltage peak will never be larger than $15 \%$.

We are going to demonstrate the approach by sizing the capacitor of the converter connected at T2. For this, we consider the converter operating as a load at nominal power, and all the power

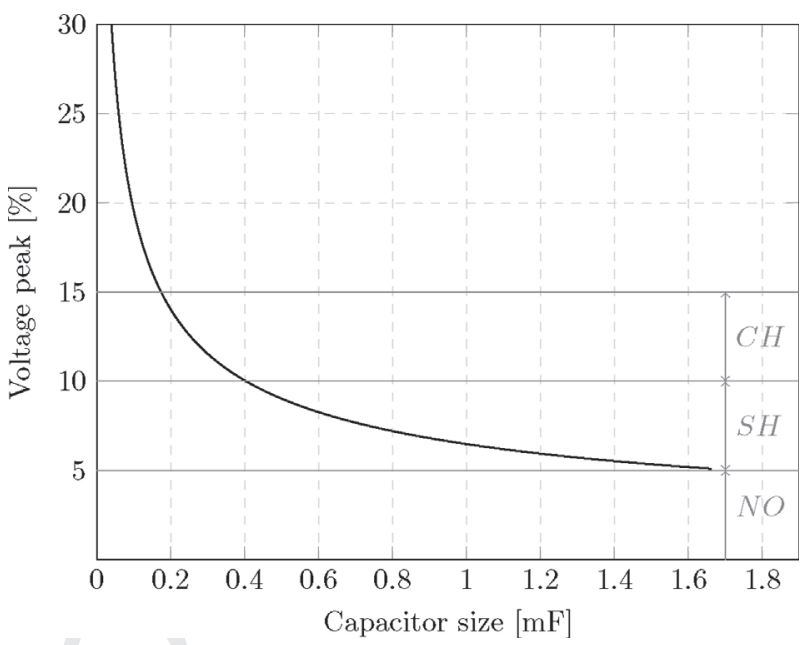

Fig. 13. Voltage overshoot at terminal 2 during load trip vs. capacitor size.

Table 4

Output capacitors of the VSCs,

\begin{tabular}{lccccc}
\hline Terminal: & T1 & T2 & T3 & T4 & T5 \\
\hline$C[\mathrm{mF}]$ & 0.075 & 0.275 & 0.075 & 0.0045 & 0.09 \\
\hline
\end{tabular}

lines linking T2 with the rest of the network connected. For this operating scenario we calculated the voltage overshoot that would occur at T2 in case of a trip for different values of $C_{2}$. These values can be seen in Fig. 13.

It can be seen that for a capacitor larger than $0.18 \mathrm{mF}$, the peak overshoot in the voltage is smaller than the $15 \%$ bound imposed by the three voltage bands. To account for the approximations made in the analysis we chose a capacitor value that gives a slightly smaller overshoot than the $15 \%$ limit. The value used in our simulation for $C_{2}$ is $0.275 \mathrm{mF}$. The others capacitors were tuned using a similar approach, and the final values used in simulation are shown in Table 4.

A rather large difference can be observed between the size of the capacitor connected at T2 and the capacitors connected at T1 and T3, despite the fact that the power ratings of the three converters are equal. This leads us to the conclusion that the size of the output capacitor is not only related to the power rating of the converter, but also to its position in the network. T1 and T3 are more strongly connected to the network, each of them being connected through rather short lines to three neighboring terminals. The converter at $\mathrm{T} 5$ on the other hand has a more isolated position; only two neighboring terminals and one of them, i.e. T1, located at a very long distance. Therefore, if a perturbation appears at T2, more capacitance is required in order to maintain the voltage inside the operating limits, and thus gain some time until the perturbation propagates to the other terminals of the network and they start to participate in the voltage regulation.

\subsection{Simulation results}

Once the size of the capacitors has been chosen, the damping controllers proposed in Section 5 can be designed for the five converters. The response of the network under classic droop control, compared with the response obtained with the damping controller can be seen in Fig. 14.

The results are obtained considering the following operating scenario: the network is at the steady state operating point shown in Table 5, with T1 loading the network, and T4 and T5 acting as 
Table 5

Steady state operating point $\wedge$

\begin{tabular}{|c|c|c|c|c|c|}
\hline Terminal: & $\mathrm{T} 1$ & $\mathrm{~T} 2$ & T3 & $\mathrm{T} 4$ & $\mathrm{~T} 5$ \\
\hline$P_{n}[\mathrm{MW}]$ & 2275.6 & -13.91 & 23.84 & -750 & -1550 \\
\hline$V_{d c}[\mathrm{p} . \mathrm{u}]$ & 0.9981 & $\wedge \quad 0.9998$ & 1.0004 & 1.0023 & 1.0023 \\
\hline
\end{tabular}

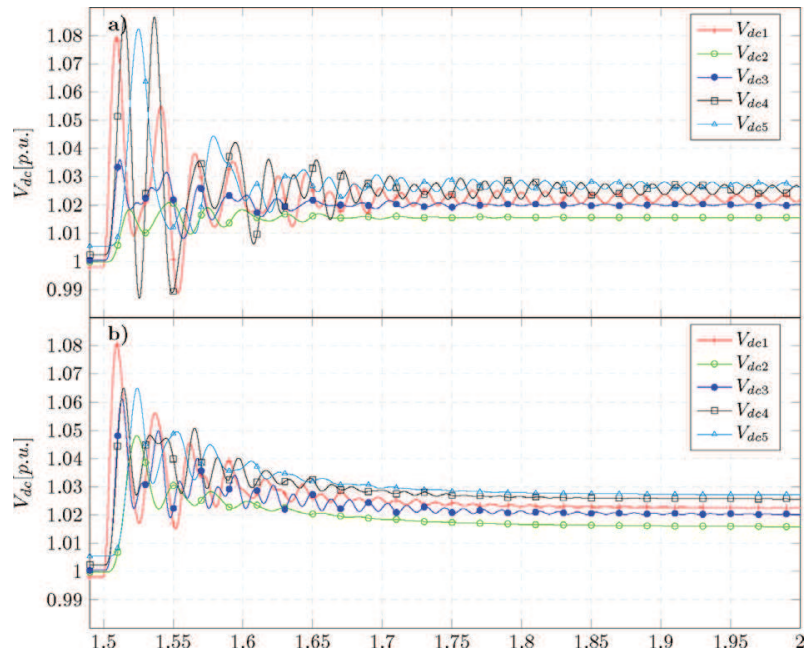

Fig. 14. Response of the dc network with $\lambda^{a}$ ) droop control and (b) droop control with dynamic damping

the main generation source. At $t=1.5$ s the load at terminal T1 trips, leaving $\mathrm{T} 2$ and $\mathrm{T} 3$ to compensate for the loss.

Fig. 14a shows the response of the network with simple droop control. The overshoot in the voltages remains in the $15 \%$ limits, and the oscillations take around 1,5 to be damped, especially at T4, due to the reduced capacitor size.

Fig. 14b shows the response of the network with damping control, and as it can be noticed, the oscillations are damped much faster. After less than 0.4 , the oscillations in all the buses are completely attenuated.

\section{Conclusions}

This paper addresses the sizing of output capacitors together with the droop control for VSCs connected to MTDC networks. For this type of networks the output capacitor of the VSC is no longer just a filtering element, but the main inertial element of the grid. Therefore, when dimensioning this element, its behavior in the scope of the network has to be taken into account. Capacitors that are too small can give rise to large voltage transients during sudden load changes, while large capacitors impose difficult requirements for the protection system.

In the work presented in this paper the capacitors are sized by analyzing their effect on the voltage peaks that appear in the network during transients. In this way, by setting a limit on the overshoot of the voltage, the capacitors can be dimensioned in order to make sure that this limit will not be over-passed.

Throughout our analysis it is shown that the values of the droop resistances used for primary control also has a strong impact on the damping of voltage transients. Therefore, an enhanced droop controller with increased damping at the oscillating frequencies is proposed and tested.

The proposed methods are validated by taking as a study case the dc grid test system proposed by the CIGRE B4 working group. Starting from the structure of this benchmark network and from the power ratings of each node, we have shown how the presented methods can be used for designing the output capacitors and the primary control in order to reduce the overshoot and the oscillations in the voltages during transients.

\section{Acknowledgements}

This work was supported in part by the Spanish Ministry Q434 of Science under Project ENE2013-48428-C02-2-R. Any opinions, findings, and conclusions or recommendations expressed in this material are those of the authors and do not necessarily reflect those of the host institutions or founders.

\section{References}

[1] IEEE, HVDC Projects Listing, Technical Report, IEEE Transmission and Distribution Committee, 2012.

[2] M. Barnes, A. Beddard, Voltage source converter HVDC links - the state of the art and issues going forward, Energy Proc ${ }_{\wedge} 24$ (0) (2012) 108-122, http://dx.doi.org/10.1016/j.egypro.2012.06.092

[3] J. Peralta, H. Saad, S. Dennetiere, J. Mahseredjian, S. Nguefeu, Detailed and averaged models for a 401-level MMC-HVDC system, IEEE Trans, Power Deliv. 27 (3) (2012) 1501-1508, http://dx.doi.org/10.1109/TPWRD.2012.2188911

[4] T.M. Haileselassie, M. Molinas, T. Undeland, Multi-terminal VSC-HVDC system for integration of offshore wind farms and green electrification of platforms in the North Sea, in: Proceedings of Nordic Workshop on Power and Industrial Electronics, Espoo, Finland, 2008, pp. 1-8.

[5] S. Cole, J. Beerten, R. Belmans, Generalized dynamic VSC MTDC model for power system stability studies, IEEE Trans, Power Syst. 25 (3) (2010) 1655-1662, http://dx.doi.org/10.1109/TPWRS.2010.2040846,

[6] O. Gomis-Bellmunt, J. Liang, J. Ekanayake, R. King, N. Jenkins, Topologies of multiterminal HVDC-VSC transmission for large offshore wind farms, Electr Power Syst. Res. 81 (2)(2011) 271-281, http://dx.doi.org/10.1016/j.epsr.2010.09.006,

[7] C. Dierckxsens, K. Srivastava, M. Reza, S. Cole, J. Beerten, R. Belmans, A distributed DC voltage control method for VSC MTDC systems, Electr Power Syst. Res. 82 (1) (2012) 54-58, http://dx.doi.org/10.1016/j.epsr.2011.08.006

[8] T.M. Haileselassie, K. Uhlen, Power system security in a meshed North Sea HVDC grid, Proc. IEEE 101 (4) (2013) 978-990, http://dx.doi.org/10.1109/JPROC.2013.2241375.

[9] M.K. Bucher, R. Wiget, G. Andersson, C.M. Franck, Multiterminal HVDC networks-what is the preferred topology? IEEE Trans. Power Deliv. 29 (1) (2014) 406-413, http://dx.doi.org/10.1109/TPWRD.2013.2277552

[10] A. Gomez-Exposito, J.M. Mauricio, J.M. Maza-Ortega, VSC-pased MVDC railway electrification system, IEEE Trans. Power Deliv. 29 (1) (2014) 422-431, http://dx.doi.org/10.1109/TPWRD.2013.2268692,

[11] K. Sun, L. Zhang, Y. Xing, J.M. Guerrero, A distributed control strategy based on DC bus signaling for modular photovoltaic generation systems with battery energy storage, IEEE Trans, Power Electron. 26 (10) (2011) 3032-3045, http://dx.doi.org/10.1109/TPEL.2011.2127488,

[12] X.-P. Zhang, Multiterminal voltage-sourced converter-based HVDC models for power flow analysis, IEEE Trans Power Syst. 19 (4) (2004) 1877-1884 http://dx.doi.org/10.1109/TPWRS.2004.836250,

[13] J. Schonberger, R. Duke, S.D. Round, DC-pus signaling: a distributed contro] strategy for a hybrid renewable nanogrid, IEEE Trans, Ind. Electron. 53 (5) (2006) 1453-1460, http://dx.doi.org/10.1109/TIE.2006.882012

[14] K. Kurohane, T. Senjyu, A. Yona, N. Urasaki, T. Goya, T. Funabashi, A hybrid smart AC/DC power system, IEEE Trans. Smart Grid 1 (2) (2010) 199-204, http://dx.doi.org/10.1109/TSG.2010.2053392

[15] K. Kurohane, A. Uehara, T. Senjyu, A. Yona, N. Urasaki, T. Funabashi, C.-H. Kim, Control strategy for a distributed DC power system with renewable energy, Renew Energy 36 (1) (2011) 42-49, http://dx.doi.org/10.1016/j.renene.2010.05.017,

[16] R.T. Pinto, P. Bauer, S.F. Rodrigues, E.J. Wiggelinkhuizen, J. Pierik, B. Ferreira, A Novel Distributed Direct-Voltage Control Strategy for Grid Integration of Offshore Wind Energy Systems Through MTDC Network, 2013, http://dx.doi.org/10.1109/TIE.2012.2216239.

[17] J.M. Guerrero, J.C. Vasquez, J. Matas, L.G. de Vicuna, M. Castilla, Hierarchical control of droop-controlled AC and DC microgrids - a general approach toward standardization, IEEE Trans Ind. Electron. 58 (1) (2011) 158-172, http://dx.doi.org/10.1109/TIE.2010.2066534

[18] K. Rouzbehi, C. Gavriluta, I. Candela, A. Luna, P. Rodriguez, Comprehensive analogy between conventional AC grids and DC grids characteristics, in: IEEE, IECON, Vienna, Austria, 2013.

[19] V. Akhmatov, M. Callavik, C.M. Franck, S.E. Rye, T. Ahndorf, M.K. Bucher, H. Muller, F. Schettler, R. Wiget, Technical guidelines and prestandardization 
work for first HVDC grids, IEEE Trans. Power Deliv. 29 (1) (2014) 327-335, http://dx.doi.org/10.1109/TPWRD.2013.2273978

[20] T.K. Vrana, J. Beerten, R. Belmans, O.B. Fosso, A classification of DC node voltage control methods for HVDC grids, Electr, Power Syst. Res. 103 (2013) 137-144, http://dx.doi.org/10.1016/j.epsr.2013.05.001 http://www.sciencedirect.com/science/article/pii/S0378779613001193

[21] T.M. Haileselassie, K. Uhlen, Impact of DC line voltage drops on power flow of MTDC using droop control, IEEE Trans, Power Syst. 27 (3) (2012) 1441-1449, http://dx.doi.org/10.1109/TPWRS.2012.2186988

[22] M. Aragüés-Pe nalba, A. Egea-Âlvarez, O. Gomis-Bellmunt, A. Sumper, Optimum voltage control for loss minimization in HVDC multi-terminal transmission systems for large offshore wind farms, Electr Power Syst. Res. 89 (2012) 54-63, http://dx.doi.org/10.1016/j.epsr.2012.02.006 http://www.sciencedirect.com/science/article/pii/S0378779612000478

[23] M. Aragüés-Pe nalba, A. Egea-Àlvarez, S.G. Arellano, O. Gomis-Bellmunt, Droop control for loss minimization in HVDC multi-terminal transmission systems for large offshore wind farms, Electr Power Syst. Res. 112 (2014) 48-55, http://dx.doi.org/10.1016/j.epsr.2014.03.013 http://www.sciencedirect.com/science/article/pii/S037877961400100X

[24] A. Egea-Alvarez, J. Beerten, D. Van Hertem, O. Gomis-Bellmunt, Primary and secondary power control of multiterminal HVDC grids, in: 10th IET International Conference on AC and DC Power Transmission (ACDC 2012), 2012, pp. 1-6, http://dx.doi.org/10.1049/cp.2012.1989.
[25] B4-58, B4-57, The CIGRE B4 DC Grid Test System, Technical Report, Cigre SC B4 HVDC and Power Electronics, 2013 http://www.b4.cigre.org/ Publications/Documents-related-to-the-development-of-HVDC-Grids

[26] R.W. Erickson, D. Maksimovic, Fundamentals of Power Electronics, Springer, 2001 http://www.books.google.es/books?id=0n9-rJTR8ygC

[27] P. Karlsson, DC Distributed Power Systems, Lund University, 2002 (Ph.D. thesis).

[28] C. Gavriluta, I. Candela, A. Luna, J. Rocabert, P. Rodriguez, Adaptive droop for primary control in MTDC networks with energy storage, in: 15th European Conference on Power Electronics and Applications (EPE), 2013, pp. 1-9, http://dx.doi.org/10.1109/EPE.2013.6631976.

[29] J. Cao, W. Du, H.F. Wang, S.Q. Bu, Minimization of transmission loss in meshed AC/DC grids with VSC-MTDC networks, IEEE Trans. Power Syst. 28 (3) (2013) 3047-3055, http://dx.doi.org/10.1109/TPWRS.2013.2241086,

[30] S. Rodrigues, R.T. Pinto, P. Bauer, J. Pierik, Optimal power flow control of VSC-based multiterminal DC network for offshore wind integration in the North Sea, JEEE J. Emerg. Select. Top. Power Electron. 1 (4) (2013) 260-268, http://dx.doi.org/10.1109/JESTPE.2013.2281917,

[31] C. Gavriluta, I.J.C. Citro, J. Rocabert, P. Rodriguez, Decentralized primary control of MTDC networks with energy storage and distributed generation, jndustry applications, IEEE Trans Ind. Appl. 99 (2014), http://dx.doi.org/10.1109/TIA.2014.2315715, 1-1. 